\title{
Endokrin Bozukluğu Olan Hastalarda Bazal Metabolik Hızın Belirlenmesinde İndirekt Kalorimetre ile Diğer Enerji Denklemlerinin Karşılaştırılması
}

\author{
Comparison of Indirect Calorimetry and Predictive Equations for Determination of Basal \\ Metabolic Rate of Patients with Endocrine Disorders
}

\author{
Özgün Tütünc $\ddot{u ̈}^{1}$, Mendane Saka ${ }^{2}$
}

Geliş tarihi/Received: 23.11.2018 • Kabul tarihi/Accepted: 12.03.2019

\section{ÖZET}

Amaç: Bu çalışma, endokrin hastalıklara sahip ve ayaktan tıbbi tedavialan hastaların bazal metabolizma hızı hesaplanmasında kullanılan enerji denklemleri ile indirekt kalorimetre sonuçlarını karşılaştırarak, bu hasta grubunun enerji gereksinmesinin belirlenmesinde en doğru sonucu veren denklemlerin belirlenmesi amacı ile yapılmıştır.

Bireyler ve Yöntem: Çalışma, Aralık 2016-Şubat 2017 ayları arasında Başkent Üniversitesi Ankara Hastanesi Endokrinoloji Bölümü'ne başvuran, 18-86 yaş arası, indirekt kalorimetre (IC) (COSMED, Fitmate GS) ile bazal enerji harcamaları ölçülen ve çalışmaya katılma konusunda gönüllü olan 150 hasta (\%74 kadın, \%26 erkek) üzerinde yapılmıştır. Bireylerin kişisel özellikleri ve yaşam tarzları anket formu ile sorgulanmıştır. Antropometrik ölçümleri ve vücut bileşimi analizleri ölçülmüş ve kaydedilmiştir. Ayrıca bireylerin antropometrik ölçümleri ve vücut bileşimleri enerji denklemlerinde kullanılarak bireylerin bazal metabolik hızları (BMH) 42 ayrı enerji denklemi ile hesaplanmıştır.

Bulgular: İndirekt kalorimetre kullanımının mümkün olmadığı durumlarda endokrin hastası bireylerin BMH'nin belirlenmesinde, tüm bireylerde Harris-Benedict (HB) 1984, erkek bireylerde Lazzer (BC), yetişkin bireylerde Nelson (BC), yaşlı bireylerde HB 1984, HB 1919 ve De Lorenzo, hafif kilolu bireylerde Henry, obez ve morbid obez bireylerde ise Huang ve Japanese (sadeleştirilmiş) denklemlerinin kullanımının en doğru sonuçları vereceği belirlenmiştir. Kadın bireyler ile zayıf ve normal bireylerin BMH'lerinin belirlenmesinde ise IC ile yeterli uyuma sahip hiçbir denklem belirlenememiştir.

Sonuç: Endokrin hastalığa sahip bireylerde IC kullanımının mümkün olmadığı durumlarda BMH’nin belirlenmesinde HB 1984 denkleminin kullanımının en doğru sonuçları vereceği belirlenmiştir.

Anahtar kelimeler: Bazal metabolik hı, indirekt kalorimetre, enerji denklemleri, endokrin hastalıklar

\section{ABSTRACT}

Aim: The purpose of this study was to specify the equations yielding the most accurate result for the determination of basal metabolic rate of outpatients with endocrine disorders by comparing the indirect calorimetry results with predictive equations.

Subjects and Method: This study was conducted on 150 voluntary patients (female 74\%, male $26 \%$ ) aged between 18 to 86 years, who admitted to Başkent University Ankara Hospital Endocrinology Department between December 2016 and February

1. İletişim/Correspondence: Başkent Üniversitesi, Sağllk Bilimleri Fakültesi, Beslenme ve Diyetetik Bölümü, Ankara, Türkiye

- E-posta: dyt.ozguntutuncu@gmail.com

(1) https://orcid.org/0000-0001-8101-1024
2. Başkent Üniversitesi, Sağlık Bilimleri Fakültesi, Beslenme ve Diyetetik Bölümü, Ankara, Türkiye (1) https://orcid.org/0000-0002-5516-426X 
2017. The basal metabolic rate (BMR) was measured by indirect calorimetry (IC) (COSMED, Fitmate GS). Demographics and information related to individual lifestyles were obtained by a questionnaire. Anthropometric and body composition analysis were measured and recorded. Furthermore, BMR was calculated with 42 different predictive equations by using the anthropometric and body composition measurements.

Results: Harris-Benedict (HB) 1984 equation was found to be the most accurate equation for determination of BMR in endocrine patients when the use of indirect calorimetry is not possible. In addition, (1) Lazzer (BC), (2) Nelson (BC), (3) HB 1984, HB 1919, De Lorenzo, (4) Henry, and (5) Huang-Japanese (simplified) gave the most accurate estimations for males, adults, elderly, overweight, and obese or morbid obese subjects, respectively. None of the BMR equations showed similar results with IC in females, and in underweight or normal weight subjects.

Conclusion: In cases where indirect calorimetry is not available, the HB 1984 equation can be used to estimate basal metabolic rates of endocrine patients.

Keywords: Basal metabolic rate, indirect calorimetry, predictive equations, endocrine disorders

\section{GíRIŞ}

Vücudun günlük enerji gereksinimi, bazal metabolizma hızı (BMH), fiziksel aktivite (FA) ve besinlerin termik etkisinin (TEF) toplamıdır. Toplam enerji harcamasının çoğunu BMH oluşturur, fiziksel aktivite ise kişiden kişiye değişmektedir (1). BMH, kişinin yaşamsal faaliyetlerini gerçekleştirebilmek için gereksinme duyduğu enerji miktarıdır. $\mathrm{BMH}$ \%60-70'i karaciğer, beyin, böbrek ve kalp gibi ana organların harcadıkları enerjiden oluşmaktadır (2). Vücut yüzeyi, cinsiyet, yaş, gebelik, kas dokusu, büyüme, endokrin hormonlar, uyku, ateş, çevre ısısı, menstürasyon durumu, hastalık durumu, katekolaminler, bazı ilaçlar ve tedavi girişimleri enerji gereksinmesini etkileyen etmenlerdir $(1,3)$.

Bazal metabolizma hızı yaklaşık 100 yıldır kullanımda olan ve yenileri türetilen enerji formülleri ile hesaplanmaktadır $(4,5)$. Bu enerji formülleri sağlıklı bireylerde doğru sonuçlar verebilmesine karşllık, yaşlı veya hasta olan bireylerde yeterince doğru sonuçlar vermemektedir $(6,7)$. Bu nedenle BMH'nin ölçümü, enerji gereksiniminin belirlenmesinde altın standart sayılmaktadır (8).

İndirekt kalorimetre (IC), solunum gazlarının değişimini analiz ederek, enerji harcanmasının ölçülmesini sağlayan invaziv olmayan bir yöntemdir. İndirekt kalorimetrenin esasları 19. yüzyılda oluşturulmuştur (9-11). Buna göre IC ölçümü uygun cihazların (sabit veya taşınabilir metabolik monitör) kullanımı ile genellikle istirahat koşullarında karbondioksit çıkışı $\left(\mathrm{VCO}_{2}\right)$ ve oksijen tüketimi $\left(\mathrm{VO}_{2}\right)$ ile değerlendirilmektedir. Ölçümden elde edilen veriler enerji tüketimi ve solunum katsayısının (RQ) hesaplanmasında kullanılmaktadır. Protein oksidasyonunun ek olarak değerlendirildiği durumlarda, IC ölçümü ile makro besin ögelerinin oksidasyonunun in vivo BMH'ye diferansiyel katkısı tahmin edilebilmektedir. İndirekt kalorimetrenin çalışma prensipleri kusursuza yakın oluşturulmuş ve enerji hesaplanmasinda kullanılan mevcut $\mathrm{BMH}$ denklemlerine göre daha doğru sonuç verdiği yapılan çalışmalarda gösterilmiştir (12-23).

Indirekt kalorimetre genellikle metabolizma hızının belirlenmesinde "altın standart” olarak kabul edilmektedir (24,25). Ancak yüksek maliyeti ve eğitimli teknik eleman gereksinmesinden dolayı IC'nin sahada kullanımı sinırlıdır. Dolayısıyla IC çoğunlukla polikliniklerde ve bilimsel araştırmalarda kullanılmaktadır. Sahada IC'ye alternatif olarak, enerji gereksinmesinin hesaplanmasında genellikle vücut ağırlığı, boy uzunluğu, yaş ve cinsiyete dayalı enerji denklemleri kullanılmaktadır. Yapılan bir derlemede, enerji denklemlerinin kullanımını doğrulayan birçok bilimsel çalışma bulunduğu belirtilmiştir (14). Ancak enerji gereksinmesinin hesaplanmasında 
en uygun ve en doğru sonucu veren denklemin ne olduğu konusunda literatürde çelişkili sonuçlar bulunmaktadır $(26,27)$. Yaş, cinsiyet, vücut bileşimi, etnik köken ile beraber metabolik stres, kas tonusu, vücut ısısı ve sakinleştirici kullanımı da dahil olmak üzere birçok etmenden etkilenmesinden dolayı BMH hesaplanmasinda kullanılan denklemlerin hedef kitlenin özelliklerine uygun olacak şekilde seçilmesi gerekmektedir (28).

$\mathrm{Bu}$ çalışma, ayaktan tedavi alan endokrin hastalarında, enerji denklemleri ile hesaplanan ve IC ile ölçülen BMH sonuçlarını karşılaştırmak ve enerji gereksinmesinin belirlenmesinde ölçüme en yakın sonucu veren BMH denklemlerini saptamak amacı ile yapılmıştır.

\section{BİREYLER VE YÖNTEM}

\section{Araştırma Yeri, Zamanı ve Örneklem Seçimi}

Çalışma, Aralık 2016-Şubat 2017 ayları arasında Başkent Üniversitesi Ankara Hastanesi Endokrinoloji Bölümü’ne başvuran, 18-86 yaş arası gönüllü bireyler ile yapılmıştır. Menstruasyon durumundaki kadınlar ile gebeler veya emzirme durumu olan hastalar çalışma dışında bırakılmıştır. Farklı enerji denklemlerinin, altın standart olan IC ile uyumlarını belirlemek amacı ile basit doğrusal regresyon uygulaması yapabilmek ve orta büyüklükte açıklama katsayısı saptayabilmek için \%95 güç ve \%5 hata olasılığı ile en az 142 hasta üzerinde çalışılmasına karar verilmiştir.

$\mathrm{Bu}$ çalışma için Başkent Üniversitesi Tıp ve Sağlık Bilimleri Araştırma Kurulu tarafından KA16/346 nolu ve 07/12/2016 tarihli “Etik Kurul Onayı” alınmıştır. Araştırmaya katılmayı kabul eden tüm bireylerden ‘Gönüllü Onam Formu’ alınmıştır.

\section{Bazal Metabolik Hızın Ölçülmesi ve Hesaplanması}

BMH, IC (COSMED, Fitmate GS) ile ölçülmüştür. Ölçümler alınırken bireylerin 12-14 saatlik açlık durumunda, dinlenme halinde, sabit ve yatar şekilde olmasına, ortamın sessiz, sabit oda sıcaklığında olmasına dikkat edilmiştir. Ölçümler 45 dakika sürmüş ve ölçüm sırasında ortamda bireyi uyaracak herhangi bir değişiklik yapılmamıştır. Bireylerin demografik özelliklerinin belirlenmesi amacı ile anket formu uygulanmıştır.

BMH hesabı hem IC hem de denklemlerle yapılmıştır. Enerji denklemleri ile BMH hesaplanması için bireylerin vücut ağırlıkları, boy uzunlukları ve vücut bileşimleri ölçülmüştür. Hastaların BMH’leri tablolarda yer alan toplamda 42 farklı enerji denklemi ile hesaplanmıştır (13,18,19,21,29-32).

\section{Verilerin İstatistiksel Değerlendirmesi}

Çalışmada elde edilen verilerin değerlendirilmesi ve tabloların oluşturulması amacıyla SPSS (Statistical Package for Social Sciences) versiyon 21.0 programı kullanılmıştır. Enerji denklemleri ile hesaplanan BMH'lerin altın standart kabul edilen IC ölçümlerini ne ölçüde açıkladığını araştırmak amacı ile 'Basit Doğrusal Regresyon’ analizi uygulanmıştır. Basit regresyon analizi sonucunda elde edilen belirlilik katsayıları $\left(\mathrm{R}^{2}\right)$ tablolarda yüzde (\%) oranları olarak sunulmuştur. Belirlilik katsayısının \%100'e yaklaşması, enerji denkleminin, IC ölçüm sonucunu ne derece iyi açıkladığı şeklinde yorumlanmıştır. Bütün istatistiksel analizlerde önemlilik düzeyi olarak $\mathrm{p}<0.05$ olarak kabul edilmiştir.

\section{BULGULAR}

\section{Bireylerin Genel Özellikleri}

Çalışmaya yaş ortalaması $54.6 \pm 16.32$ yıl olan toplam 150 kişi (\%26 erkek) dahil edilmiştir. Bireylerin \%66'sı 18-64 yaş aralığındadır. Çalışmaya katılan bireylerin \%51.3’ü diyabet - insülin direnci, \%37.3’ü hipertansiyon, \%80’i tiroid hastalığı, \%26.7'si obezite, \%60’ı lipit bozukluğu teşhisi almıştır. Çalışmaya katılan bireylerin BKİ ortanca değeri $27.8 \mathrm{~kg} / \mathrm{m}^{2}$ 'dir. Dünya Sağlık Örgütü'nün BKİ sinıflamasına göre bireylerin \%30’u normal, \%42.3'ü hafif şişman, \%24’ü obez, \%3.3'ü ise morbid obez sınıfında bulunmaktadır (tabloda gösterilmemiş veri). 
İndirekt Kalorimetre ölçümü ve Enerji Denklemlerine göre Hesaplanan Bazal Metabolik Hızlarının Regresyon Analizi Yardımı ile Karşılaştırılması

Çalışmaya katılan bireylerin enerji denklemleri ile hesaplanan BMH'nin, IC ile ölçülen BMH'yi açıklama oranları ve önemlilik düzeyleri Tablo 1, Tablo 2 ve Tablo 3'te sunulmuştur. Bu tablolarda aynı zamanda tüm bireylerin IC ve enerji denklemlerinden elde edilen $\mathrm{BMH}$ ortalama ve standart sapma değerleri de gösterilmiştir.

Endokrin hastalı̆ga sahip tüm bireylerde HarrisBenedict (HB) 1984, IC ile en yüksek uyuma sahip olan enerji denklemi olarak belirlenmiştir (Tablo 1).

Çalışmaya katılan bireyler cinsiyetlerine göre gruplandırıldığında erkek bireylerde Lazzer (BC) denkleminin IC ile en yüksek uyuma sahip olduğu belirlenmişken kadınlarda IC ile yeterli uyuma sahip herhangi bir enerji denklemi belirlenememiştir (Tablo 2).

Bireyler yaş gruplarına göre ayrıldığında, yetişkin bireylerde Nelson (BC), yaşlı bireylerde HB 1984, HB 1919 ve De Lorenzo denklemleri IC ile en yüksek uyuma sahip olan denklemler olarak belirlenmiş̧ir (Tablo 3).

Çalışmaya katılan bireylerin verileri BKİ gruplarına göre ayrıldığında, hafif kilolu bireylerde Henry, obez ve morbid obez bireylerde ise Huang ve Japanese (sadeleştirilmiş) denklemlerinin IC ile en uyumlu sonuçlara sahip olduğu belirlenmiştir. Zayıf ve normal bireylerin BMH'lerinin belirlenmesinde IC ile yeterli uyuma sahip bir denklem belirlenememiştir (tabloda gösterilmemiş veri).

\section{TARTIŞMA}

BMH'nin, IC ile ölçülmesi, enerji gereksiniminin belirlenmesinde altın standart olarak kabul edilmektedir (8). Ancak yüksek maliyeti ve eğitimli teknik eleman gereksinmesi nedeniyle IC'nin sahada kullanımı sınırlıdır (14). Bu nedenle klinik pratikte, BMH'nin hesaplanması için oluşturulan çeşitli denklemler kullanılmaktadır. BMH'nin enerji formülleri ile hesaplanması sağlıklı bireylerde doğru sonuçlar verebilmesine karşılık, yaşlılık veya hastalık olması durumunda doğru sonuçlar vermemektedir (6,7). Benzer şekilde endokrin hastalığa sahip bireylerin metabolik yaşlarının ve vücut bileşimlerinin sağlıklı bireylerden farklı olması, antropometrik ölçümlere ve vücut bileşimine dayalı enerji denklemlerinin bu kişilerde yanlış sonuçlar vermesine neden olabilmektedir (14,33-35).

Endokrin hastalığı olan bireylerde, IC ile ölçülen BMH ile 42 farklı enerji denkleminden elde edilen sonuçların karşlaştırıldığ bu çalışmada tüm bireylerde regresyon analizi sonucunda IC ile ölçülen BMH'yi en iyi açıklayan ilk üç denklem Bernstein (BC) (\%67.7), Henry (\%67.4) ve HB 1984 denklemi (\%67.2) olarak belirlenmiştir. Bu hasta grubunda IC kullanımının mümkün olmadığ durumlarda, bu denklemlerin kullanımının en doğru sonuçları verdiğini, ancak çalışmaya dahil edilen denklemlerin hiçbirinin IC yerine kullanılamayacağ belirlenmiştir. Benzer şekilde, Nagano et al. (30), serebral infarkt teşhisi ile hastanede yatan 45-96 yaş aralığında, 30 hasta üzerinde IC ölçümü ile 6 farklı enerji denklemini karşılaştırdıkları çalışmada HB denkleminin en düşük standart sapmaya sahip olduğu ve bu denklemi Cunningham, Wang, Mifflin, Japanese (sadeleştirilmiş) ve Owen denklemlerinin izlendiği saptanmıştır. Araştırmacılar ayrıca malnütrisyon riski olan hastalarda IC sonuçlarına göre yüksek sonuç veren Cunningham ve Wang denklemlerinin, malnütrisyon riski olmayan hastalarda ise HB denkleminin daha uygun olacağını bildirmişlerdir (30). Boullata et al. (21) hastanede yatan 16-92 yaş arasında 395 hasta üzerinde IC ile ölçülen BMH ile 8 farklı enerji denkleminden elde edilen BMH'leri karşılaş̧ırdıkları çalışmada 1.1 stres faktörü ile HB, Mifflin, Ireton-Jones 1992, ACCP önerileri, Ireton-Jones obezite, Hamwi $\times 1.3$, James $\times 1.3$, Swinamer ve Penn-State denklemlerine klyasla IC ile en yüksek uyumu gösteren enerji denklemi olarak belirlemiş, ancak bu uyumun istatistiksel olarak kabul edilemeyeceğini belirtilmiştir. 
Tablo 1. Bireylerin indirekt kalorimetre ile ölçülmüş bazal metabolik hızları ile enerji denklemlerinden elde edilen bazal metabolik hızlarının basit doğrusal regresyon $\left(\mathrm{R}^{2}\right)$ yöntemiyle açıklanması

\begin{tabular}{|c|c|c|c|}
\hline Enerji denklemleri & BMH (kkal/gün) $\bar{X}_{ \pm}$SS & $\begin{array}{c}\mathbf{R}^{2} \\
(\%)\end{array}$ & $\mathbf{p}$ \\
\hline İndirekt kalorimetre & $1421.2 \pm 290.19$ & - & - \\
\hline Bernstein (vücut bileşim) & $1187.2 \pm 208.58$ & 67.7 & $0.000^{\circ}$ \\
\hline Henry & $1457.1 \pm 249.75$ & 67.4 & $0.000^{\circ}$ \\
\hline HB 1984 & $1499.3 \pm 253.93$ & 67.2 & $0.000^{\circ}$ \\
\hline HB 1919 & $1481.6 \pm 261.27$ & 66.8 & $0.000^{\circ}$ \\
\hline De Lorenzo & $1504.0 \pm 264.30$ & 66.8 & $0.000^{\circ}$ \\
\hline Lazzer & $1558.8 \pm 254.03$ & 66.8 & $0.000^{\circ}$ \\
\hline Henry (yaş) & $1463.3 \pm 263.98$ & 66.4 & $0.000^{\circ}$ \\
\hline Huang & $1467.1 \pm 271.27$ & 65.8 & $0.000^{\circ}$ \\
\hline Black & $1452.1 \pm 240.27$ & 65.5 & $0.000^{\circ}$ \\
\hline Livingston & $1406.8 \pm 236.34$ & 65.0 & $0.000^{\circ}$ \\
\hline Mifflin & $1387.5 \pm 262.53$ & 64.9 & $0.000^{\circ}$ \\
\hline WHO/FAO/UNU 1985 (vücut ağırlığı) & $1512.7 \pm 256.31$ & 64.7 & $0.000^{\circ}$ \\
\hline FAO (yaş), 2004 & $1512.7 \pm 256.31$ & 64.7 & $0.000^{\circ}$ \\
\hline Bernstein & $1213.8 \pm 202.47$ & 64.6 & $0.000^{\circ}$ \\
\hline Japanese (sadeleştirilmiş) & $1507.6 \pm 269.68$ & 64.6 & $0.000^{\circ}$ \\
\hline FAO & $1511.9 \pm 242.35$ & 64.5 & $0.000^{\circ}$ \\
\hline Wang (vücut bileşimi) & $1466.1 \pm 216.28$ & 64.2 & $0.000^{\circ}$ \\
\hline Cunningham (vücut bileşimi) & $1434.0 \pm 217.38$ & 64.1 & $0.000^{\circ}$ \\
\hline Korth (vücut bileşimi) & $1566.6 \pm 260.08$ & 64.0 & $0.000^{\circ}$ \\
\hline Liu & $1517.6 \pm 286.17$ & 63.4 & $0.000^{\circ}$ \\
\hline Owen & $1426.4 \pm 235.07$ & 62.9 & $0.000^{\circ}$ \\
\hline Schofield (yaş, cinsiyet) & $1483.5 \pm 254.76$ & 62.6 & $0.000^{\circ}$ \\
\hline World Schofield & $1482.4 \pm 254.62$ & 62.6 & $0.000^{\circ}$ \\
\hline Mifflin (vücut bileşimi) & $1385.4 \pm 197.07$ & 62.6 & $0.000^{\circ}$ \\
\hline Owen (vücut bileşimi) & $1335.6 \pm 247.12$ & 61.4 & $0.000^{\circ}$ \\
\hline Schofield & $1515.6 \pm 279.17$ & 60.8 & $0.000^{\circ}$ \\
\hline Huang (vücut bileşimi) & $1201.4 \pm 231.51$ & 56.0 & $0.000^{\circ}$ \\
\hline Müller & $1866.3 \pm 257.42$ & 55.1 & $0.000^{\circ}$ \\
\hline ESICM’98 Bildirisi & $1855.4 \pm 570.73$ & 55.1 & $0.000^{\circ}$ \\
\hline Müller (beden kütle indeksi) & $1855.0 \pm 276.09$ & 50.6 & $0.000^{\circ}$ \\
\hline Müller (vücut bileşimi) & $1773.0 \pm 274.75$ & 44.6 & $0.000^{\circ}$ \\
\hline Ireton-Jones 1992 & $1829.2 \pm 234.81$ & 43.1 & $0.000^{\circ}$ \\
\hline Ireton-Jones Obezite & $1186.8 \pm 594.36$ & 42.1 & $0.000^{\circ}$ \\
\hline Ireton-Jones 1997 & $1623.9 \pm 236.40$ & 38.6 & $0.000^{\circ}$ \\
\hline ACCP Önerileri & $1636.2 \pm 240.28$ & 33.7 & $0.000^{\circ}$ \\
\hline WHO/FAO/UNU 1985 (vücut ağırlığı, boy) & $1412.7 \pm 352.25$ & 17.0 & $0.000^{\circ}$ \\
\hline Müller (beden kütle indeksi, vücut bileşimi) & $2661.9 \pm 941.08$ & 7.3 & $0.001^{\circ}$ \\
\hline Ireton-Jones Spontane solunum & $1810.9 \pm 923.46$ & 6.4 & $0.002^{2}$ \\
\hline Korth & $1589.3 \pm 865.13$ & 3.7 & $0.018^{\sim}$ \\
\hline Johnstone (vücut bileşimi) & $1553.9 \pm 825.76$ & 2.0 & 0.082 \\
\hline Nelson (vücut bileşimi) & $1457.4 \pm 1017.55$ & 1.7 & 0.112 \\
\hline Lazzer (vücut bileşimi) & $1212.8 \pm 361.53$ & 1.7 & 0.112 \\
\hline
\end{tabular}

BMH: Bazal metabolik hiz, HB:Harris-Benedict, ACCP: American College of Chest Physicians, ESICM: European Society of Intensive Care Medicine, WHO: World Health Organization, FAO: Food and Agriculture Organization, UNU: United Nations University, $R^{2}$ : Belirleme katsayısı. $p<0.05$. 
Tablo 2. Bireylerin cinsiyet gruplarında indirekt kalorimetre ile ölçülmüş bazal metabolik hızlarının, enerji denklemlerinden elde edilen bazal metabolik hızlar ile basit doğrusal regresyon $\left(\mathrm{R}^{2}\right)$ yöntemi ile açıklanması

\begin{tabular}{|c|c|c|c|c|c|c|}
\hline \multirow{2}{*}{$\begin{array}{l}\text { Indirekt kalorimetre ve enerji } \\
\text { denklemleri }\end{array}$} & \multicolumn{3}{|c|}{ Erkek $(n=39)$} & \multicolumn{3}{|c|}{ Kadın $(n=111)$} \\
\hline & $\begin{array}{c}\text { BMH (kkal/gün) } \\
\bar{X} \pm S S\end{array}$ & $\begin{array}{c}\mathbf{R}^{2} \\
(\%)\end{array}$ & $\mathbf{p}$ & $\begin{array}{c}\text { BMH (kkal/gün) } \\
\bar{X}_{ \pm S S}\end{array}$ & $\begin{array}{c}\mathbf{R}^{2} \\
(\%)\end{array}$ & $\mathbf{p}$ \\
\hline HB 1919 & $1761.9 \pm 283.41$ & 65.6 & $0.000^{-}$ & $1761.9 \pm 283.41$ & 65.6 & $0.000^{\circ}$ \\
\hline HB 1984 & $1776.8 \pm 267.42$ & 66.5 & $0.000^{-}$ & $1776.8 \pm 267.42$ & 66.5 & $0.000^{-}$ \\
\hline Black & $1742.1 \pm 206.00$ & 62.8 & $0.000^{-}$ & $1742.1 \pm 206.00$ & 62.8 & $0.000^{\circ}$ \\
\hline Owen & $1761.8 \pm 160.59$ & 60.9 & $0.000^{\sim}$ & $1761.8 \pm 160.59$ & 60.9 & $0.000^{\circ}$ \\
\hline Owen (vücut bileşimi) & $1698.5 \pm 174.75$ & 57.2 & $0.000^{\smile}$ & $1698.5 \pm 174.75$ & 57.2 & $0.000^{-}$ \\
\hline Mifflin & $1687.4 \pm 219.99$ & 65.1 & $0.000^{-}$ & $1687.4 \pm 219.99$ & 65.1 & $0.000^{-}$ \\
\hline Korth & $1915.0 \pm 239.07$ & 59.7 & $0.000^{-}$ & $1915.0 \pm 239.07$ & 59.7 & $0.000^{\circ}$ \\
\hline Korth (vücut bileşimi) & $1926.1 \pm 202.46$ & 57.2 & $0.000^{-}$ & $1926.1 \pm 202.46$ & 57.2 & $0.000^{-}$ \\
\hline De Lorenzo & $1782.4 \pm 259.19$ & 66.2 & $0.000^{-}$ & $1782.4 \pm 259.19$ & 66.2 & $0.000^{-}$ \\
\hline Lazzer & $1822.2 \pm 267.65$ & 64.6 & $0.000^{\circ}$ & $1822.2 \pm 267.65$ & 64.6 & $0.000^{\circ}$ \\
\hline Lazzer (vücut bileşimi) & $1719.1 \pm 264.93$ & 66.8 & $0.000^{\smile}$ & $1719.1 \pm 264.93$ & 66.8 & $0.000^{\circ}$ \\
\hline Huang & $1825.1 \pm 183.71$ & 66.3 & $0.000^{\smile}$ & $1825.1 \pm 183.71$ & 66.3 & $0.000^{-}$ \\
\hline Huang (vücut bileşimi) & $1560.1 \pm 123.26$ & 56.5 & $0.000^{-}$ & $1560.1 \pm 123.26$ & 56.5 & $0.000^{-}$ \\
\hline Johnstone (vücut bileşimi) & $1761.5 \pm 238.98$ & 65.5 & $0.000^{\sim}$ & $1761.5 \pm 238.98$ & 65.5 & $0.000^{-}$ \\
\hline Japanese (sadeleştirilmiş) & $1839.0 \pm 221.99$ & 60.9 & $0.000^{\circ}$ & $1839.0 \pm 221.99$ & 60.9 & $0.000^{\circ}$ \\
\hline Ireton-Jones Spontane solunum & $2025.3 \pm 381.45$ & 43.2 & $0.000^{\circ}$ & $2025.3 \pm 381.45$ & 43.2 & $0.000^{\circ}$ \\
\hline Nelson (vücut bileşimi) & $1723.6 \pm 227.52$ & 61.7 & $0.000^{-}$ & $1723.6 \pm 227.52$ & 61.7 & $0.000^{\circ}$ \\
\hline World Schofield & $1754.1 \pm 254.03$ & 62.0 & $0.000^{\sim}$ & $1754.1 \pm 254.03$ & 62.0 & $0.000^{\circ}$ \\
\hline Schofield & $1887.7 \pm 188.15$ & 54.7 & $0.000^{-}$ & $1887.7 \pm 188.15$ & 54.7 & $0.000^{-}$ \\
\hline FAO (yaş) & $1785.8 \pm 251.48$ & 65.4 & $0.000^{-}$ & $1785.8 \pm 251.48$ & 65.4 & $0.000^{-}$ \\
\hline FAO & $1770.8 \pm 235.23$ & 59.6 & $0.000^{\circ}$ & $1770.8 \pm 235.23$ & 59.6 & $0.000^{-}$ \\
\hline Henry (yaş) & $1764.1 \pm 244.46$ & 67.0 & $0.000^{-}$ & $1764.1 \pm 244.46$ & 67.0 & $0.000^{-}$ \\
\hline Henry & $1752.7 \pm 229.58$ & 67.2 & $0.000^{-}$ & $1752.7 \pm 229.58$ & 67.2 & $0.000^{-}$ \\
\hline Müller (beden kütle indeksi) & $2144.5 \pm 234.33$ & 40.0 & $0.000^{-}$ & $2144.5 \pm 234.33$ & 40.0 & $0.000^{\circ}$ \\
\hline Müller (beden kütle indeksi, vücut bileşimi) & $2914.5 \pm 953.03$ & 0.1 & 0.885 & $2914.5 \pm 953.03$ & 0.1 & 0.885 \\
\hline ACCP önerileri & $1876.4 \pm 167.28$ & 19.3 & $0.005^{-}$ & $1876.4 \pm 167.28$ & 19.3 & $0.005^{-}$ \\
\hline ESICM’98 Bildirisi & $2627.7 \pm 494.33$ & 55.0 & $0.000^{-}$ & $2627.7 \pm 494.33$ & 55.0 & $0.000^{-}$ \\
\hline Schofield (yaş, cinsiyet) & $1755.2 \pm 254.19$ & 62.0 & $0.000^{\smile}$ & $1755.2 \pm 254.19$ & 62.0 & $0.000^{\circ}$ \\
\hline WHO/FAO/UNU 1985 (vücut ağırlığı) & $1785.8 \pm 251.48$ & 65.4 & $0.000^{-}$ & $1785.8 \pm 251.48$ & 65.4 & $0.000^{\circ}$ \\
\hline WHO/FAO/UNU 1985 (vücut ağırlığı, boy) & $1389.2 \pm 635.42$ & 31.3 & $0.000^{-}$ & $1389.2 \pm 635.42$ & 31.3 & $0.000^{\circ}$ \\
\hline
\end{tabular}

$R^{2}$ : Belirleme katsayıs, BMH: Bazal metabolik hiz, ACCP: American College of Chest Physicians, ESICM: European Society of Intensive Care Medicine, WHO: World Health Organization, FAO: Food and Agriculture Organization, UNU: United Nations University, $R^{2}$ : Belirleme katsaylsi. ‘ $p<0.05$. 
Tablo 3. Bireylerin yaş gruplarına göre indirekt kalorimetre ile ölçülmüş bazal metabolik hızlarının, enerji denklemlerinden elde edilen bazal metabolik hızlar ile basit doğrusal regresyon $\left(\mathrm{R}^{2}\right)$ yöntemi ile açıklanması

\begin{tabular}{|c|c|c|c|c|c|c|}
\hline \multirow{2}{*}{$\begin{array}{l}\text { İndirekt kalorimetre ve enerji } \\
\text { denklemleri }\end{array}$} & \multicolumn{3}{|c|}{ 19-64 yaş } & \multicolumn{3}{|c|}{$65-86$ yaş } \\
\hline & $\begin{array}{c}\text { BMH (kkal/gün) } \\
\bar{X}_{ \pm S S}\end{array}$ & $\begin{array}{c}\mathbf{R}^{2} \\
(\%)\end{array}$ & $\mathbf{p}$ & $\begin{array}{c}\text { BMH (kkal/gün) } \\
\bar{X}_{ \pm S S}\end{array}$ & $\begin{array}{c}\mathbf{R}^{2} \\
(\%)\end{array}$ & $\mathbf{p}$ \\
\hline İndirekt kalorimetre ölçümü & $1452.0 \pm 287.12$ & - & - & $1361.3 \pm 289.54$ & - & - \\
\hline Harris-Benedict 1919 & $1531.4 \pm 267.84$ & 68.6 & $0.000^{-}$ & $1385.1 \pm 220.01$ & 63.6 & $0.000^{\circ}$ \\
\hline Harris-Benedict 1984 & $1545.1 \pm 258.67$ & 68.7 & $0.000^{-}$ & $1410.4 \pm 220.94$ & 63.8 & $0.000^{\circ}$ \\
\hline Bernstein & $1251.7 \pm 213.01$ & 67.7 & $0.000^{\smile}$ & $1140.3 \pm 157.76$ & 59.6 & $0.000^{-}$ \\
\hline Bernstein (vücut bileşimi) & $1209.4 \pm 214.04$ & 71.0 & $0.000^{-}$ & $1144.0 \pm 192.29$ & 59.3 & $0.000^{-}$ \\
\hline Black & $1486.3 \pm 248.18$ & 67.8 & $0.000^{-}$ & $1385.7 \pm 210.94$ & 59.1 & $0.000^{-}$ \\
\hline Owen & $1424.9 \pm 240.50$ & 71.2 & $0.000^{-}$ & $1429.3 \pm 226.47$ & 52.0 & $0.000^{-}$ \\
\hline Owen (vücut bileşimi) & $1344.1 \pm 254.78$ & 67.6 & $0.000^{\smile}$ & $1319.0 \pm 233.10$ & 50.5 & $0.000^{-}$ \\
\hline Mifflin & $1436.3 \pm 260.45$ & 66.5 & $0.000^{-}$ & $1293.0 \pm 242.09$ & 60.7 & $0.000^{\circ}$ \\
\hline Mifflin (vücut bileşimi) & $1392.8 \pm 204.44$ & 69.4 & $0.000^{-}$ & $1370.8 \pm 182.98$ & 50.5 & $0.000^{-}$ \\
\hline Livingston & $1451.4 \pm 237.56$ & 67.8 & $0.000^{-}$ & $1320.3 \pm 210.38$ & 58.6 & $0.000^{\circ}$ \\
\hline Müller & $1836.5 \pm 266.84$ & 66.3 & $0.000^{-}$ & $1924.2 \pm 229.66$ & 52.8 & $0.000^{-}$ \\
\hline Müller (vücut bileşimi) & $1747.3 \pm 293.32$ & 50.4 & $0.000^{-}$ & $1823.0 \pm 229.06$ & 48.3 & $0.000^{\circ}$ \\
\hline Korth & $1569.4 \pm 310.56$ & 65.5 & $0.000^{\smile}$ & $1627.9 \pm 1427.96$ & 0.0 & 0.907 \\
\hline Korth (vücut bileşimi) & $1578.9 \pm 268.18$ & 69.3 & $0.000^{\smile}$ & $1542.7 \pm 244.39$ & 54.1 & $0.000^{-}$ \\
\hline De Lorenzo & $1548.3 \pm 270.78$ & 68.3 & $0.000^{-}$ & $1418.0 \pm 230.30$ & 62.9 & $0.000^{-}$ \\
\hline Lazzer & $1582.0 \pm 270.97$ & 69.2 & $0.000^{-}$ & $1513.9 \pm 212.76$ & 62.3 & $0.000^{-}$ \\
\hline Lazzer (vücut bileşimi) & $1248.8 \pm 331.73$ & 2.6 & 0.113 & $1142.9 \pm 407.77$ & 2.1 & 0.306 \\
\hline Huang & $1482.0 \pm 27.68$ & 71.1 & $0.000^{-}$ & $1438.1 \pm 260.67$ & 55.8 & $0.000^{-}$ \\
\hline Huang (vücut bileşimi) & $1217.3 \pm 234.26$ & 61.9 & $0.000^{-}$ & $1170.5 \pm 225.12$ & 44.2 & $0.000^{-}$ \\
\hline Johnstone (vücut bileşimi) & $1522.5 \pm 262.60$ & 69.4 & $0.000^{\complement}$ & $1615.0 \pm 1375.17$ & 0.2 & 0.785 \\
\hline Japanese (sadeleştirilmiş) & $1507.2 \pm 281.32$ & 70.7 & $0.000^{-}$ & $1508.3 \pm 248.20$ & 57.2 & $0.000^{-}$ \\
\hline Wang (vücut bileşimi) & $1479.4 \pm 223.13$ & 69.4 & $0.000^{-}$ & $1446.3 \pm 202.99$ & 54.4 & $0.000^{-}$ \\
\hline Cunningham (vücut bileşimi) & $1444.3 \pm 224.17$ & 69.4 & $0.000^{\smile}$ & $1413.9 \pm 204.20$ & 54.1 & $0.000^{-}$ \\
\hline Ireton-Jones 1992 & $1912.1 \pm 215.33$ & 43.9 & $0.000^{-}$ & $1668.3 \pm 182.84$ & 51.8 & $0.000^{-}$ \\
\hline Ireton-Jones 1997 & $1716.0 \pm 212.02$ & 38.7 & $0.000^{-}$ & $1445.1 \pm 170.33$ & 53.9 & $0.000^{-}$ \\
\hline Ireton-Jones Obezite & $1272.0 \pm 585.15$ & 45.4 & $0.000^{-}$ & $1021.5 \pm 582.50$ & 32.7 & $0.000^{-}$ \\
\hline Liu & $1552.1 \pm 291.65$ & 66.3 & $0.000^{\smile}$ & $1450.7 \pm 265.35$ & 55.5 & $0.000^{-}$ \\
\hline Ireton-Jones spontane solunum & $1841.0 \pm 360.96$ & 35.3 & $0.000^{-}$ & $1752.4 \pm 1510.18$ & 3.0 & 0.226 \\
\hline Nelson (vücut bileşimi) & $1389.7 \pm 283.30$ & 72.3 & $0.000^{-}$ & $1589.0 \pm 1703.37$ & 0.0 & 0.901 \\
\hline World Schofield & $1535.1 \pm 271.28$ & 66.5 & $0.000^{-}$ & $1380.2 \pm 181.07$ & 58.9 & $0.000^{-}$ \\
\hline Schofield & $1538.7 \pm 271.14$ & 66.9 & $0.000^{-}$ & $1470.7 \pm 291.60$ & 49.4 & $0.000^{-}$ \\
\hline FAO (yaş) & $1559.2 \pm 270.14$ & 68.0 & $0.000^{\complement}$ & $1422.5 \pm 200.39$ & 58.9 & $0.000^{\circ}$ \\
\hline FAO & $1550.1 \pm 256.17$ & 67.3 & $0.000^{-}$ & $1437.9 \pm 194.79$ & 58.8 & $0.000^{-}$ \\
\hline Henry (yaş) & $1500.6 \pm 277.16$ & 69.6 & $0.000^{-}$ & $1390.8 \pm 221.32$ & 58.3 & $0.000^{-}$ \\
\hline Henry & $1489.7 \pm 258.52$ & 70.5 & $0.000^{-}$ & $1384.8 \pm 217.89$ & 59.6 & $0.000^{-}$ \\
\hline Müller (beden kütle indeksi) & $1838.6 \pm 287.05$ & 65.6 & $0.000^{-}$ & $1886.7 \pm 253.17$ & 32.6 & $0.000^{-}$ \\
\hline Müller (beden kütle indeksi, vücut bileşimi) & $2554.3 \pm 820.0$ & 12.0 & $0.000^{-}$ & $2870.6 \pm 1119.79$ & 6.0 & 0.083 \\
\hline ACCP önerileri & $1610.1 \pm 246.84$ & 38.5 & $0.000^{-}$ & $1686.8 \pm 220.61$ & 37.5 & $0.000^{-}$ \\
\hline ESICM’98 Bildirisi & $1975.6 \pm 586.17$ & 60.8 & $0.000^{-}$ & $1622.0 \pm 476.18$ & 42.7 & $0.000^{\circ}$ \\
\hline Schofield (yaş, cinsiyet) & $1536.2 \pm 271.40$ & 66.5 & $0.000^{-}$ & $1381.1 \pm 181.19$ & 58.9 & $0.000^{-}$ \\
\hline WHO/FAO/UNU 1985 (vücut ağırlığı) & $1559.2 \pm 270.14$ & 68.0 & $0.000^{-}$ & $1422.5 \pm 200.39$ & 58.9 & $0.000^{-}$ \\
\hline WHO/FAO/UNU 1985 (vücut ağırlığı, boy) & $1534.7 \pm 303.66$ & 48.8 & $0.000^{-}$ & $1175.9 \pm 319.97$ & 2.9 & 0.232 \\
\hline
\end{tabular}


BKİ $\leq 50.0 \mathrm{~kg} / \mathrm{m}^{2}$ olan, sağlıklı ya da kontrollü metabolik hastallklara sahip, herhangi bir ilaç tedavisi almayan, 68 yaşlı birey üzerinde IC ile ölçülmüş BMH ile 14 farklı enerji denkleminden elde edilen BMH'lerin karşılaştırıldığı başka bir çalışmada hata payı en küçük olan enerji denkleminin Müller, en büyük olanın ise Mifflin olduğu, Müller, HB ve Fredrix denklemlerinin IC'den sapma oranının hastaların \%60'ı için kabul edilebilir düzeyde olduğu bildirilmiştir (36). Hemodiyalize giren 65-74 yaş aralığındaki 72 yaşlı hasta üzerinde IC ile ölçülen BMH ile HB, Schofield ve WHO 1985 denklemlerinden elde edilen BMH'lerin karşılaştırıldığı bir başka çalışmanın sonucunda tüm enerji denklemlerinden elde edilen BMH'lerin IC sonuçlarından anlamlı düzeyde yüksek sonuçlar verdikleri saptanmıştır (29). Bu çalışmada ise, yaşlı bireylerde regresyon analizi sonucunda IC ölçümünü en iyi açıllayan üç denklem HB 1984 (\%63.8), HB 1919 (\%63.6) ve De Lorenzo (\%62.9) yetişkin bireylerde Nelson (BC) (\%72.3) denklemi olarak belirlenmiştir.

Çalışmaya katılan bireylerin sonuçları BKİ gruplarına ayrıldığında, obez ve morbid obez bireylerde regresyon analizi sonucunda IC ölçümünü en iyi açıklayan üç denklemin Huang (\%63.4), Japanese (sadeleştirilmiş) (\%62.3) ve Owen (\%61.5) olarak belirlenmiştir.Zayıf ve normal bireylerde ise regresyon analizi sonucunda IC ölçümünü en iyi açıklayan Lazzer (\%42.2), De Lorenzo (\%38.4) ve Liu (\%38.8) olarak bulunmuştur. Ancak bu denklemlerin hiç biri zayıf ve normal grupta BMH'nin belirlenmesinde IC ile yeterli uyuma sahip olamamıştır. ChungKang et al. (18), 80 obez (BKI $\geq 30.0 \mathrm{~kg} / \mathrm{m}^{2}$ ) Tayvanlı yetişkin (18-64 yaş) birey üzerinde IC ile ölçülen ve 8 farklı enerji denkleminden hesaplanan BMH'leri karşılaştırdıkları çalışmada ise IC ile en yüksek uyuma sahip denklemlerin sirasıyla Mifflin (\%46.3), Owen (\%38.8), HB (\%37.5), Schofield, Bernstein ve Cunningham denklemleri (hepsi için \%36.3), Wang (\%35.0) ve Liu (\%31.3) denklemleri olduğu ancak hiçbir denklemin IC ile yeterince yüksek uyuma sahip olmadığı saptanmıştır. Yoğun bakım servisinde yatan 18 yaşından büyük, 36 obez $\left(B K I ̇ 30 \mathrm{~kg} / \mathrm{m}^{2}\right)$ hasta üzerinde IC ile ölçülmüş BMH ile 4 farklı enerji denkleminden elde edilen enerjilerin karşılaştırıldığı başka bir çalışmada ideal ağırlığın, 1.5 stres faktörü ile birlikte HB denkleminde kullanılması ile IC'den elde edilen $\mathrm{BMH}$ ile en yüksek uyumun elde edildiği belirlenmiştir ( $r=0.59$ ) (37). Bunu düzeltilmiş ağırlığın kullanıldığı, 1.5 stres faktörü ile HB ( $\mathrm{r}=0.57)$ ve stres faktörsüz düzeltilmiş ağırlığın kullanıldığı HB (r=0.51) denklemlerinin izlendiği gözlemlenmiştir (37).

Sonuç olarak, endokrin hastalığa sahip bireylerde IC kullanımının mümkün olmadığı durumlarda BMH'nin belirlenmesinde HB 1984 denkleminin kullanımının en doğru sonuçları vereceği belirlenmiştir. Bu çalışmanın güçlü yönü endokrin hastalarında $\mathrm{BMH}$ hesabında kullanılan denklemlerin IC ölçümü ile kıyaslanmasının yapıldığı ve IC kullanımının mümkün olmadığı durumlarda bu hasta grubunda kullanılabilecek en doğru enerji denkleminin belirlendiği ilk çalışma olmasıdır. $\mathrm{Bu}$ çalışma sonuçları ile daha önce yapılan çalışmalar arasındaki farklılıklar çalışmalara dahil edilen örneklemlerin farklı hastalıklara, farklı koşullara (ayaktan tedavi alan, yatan hastalar, kritik hastalar vb.), farklı beslenme yolları ile ırksal farklılıklara sahip olmaları ve çalışmalara farklı enerji denklemlerinin dahil edilmesinden kaynaklandığı düşünülmektedir.

Çıkar çatışması - Conflict of interest: Yazarlar çıkar çatışması olmadığını beyan ederler. - The authors declare that they have no conflict of interest.

Teşekkür - Acknowledgement: Çalışmamın istatistiksel değerlendirilmesinde yardımcı olan Prof. Dr. Mehtap AKCIL OK'a ve çalışmaya katılan vakaları bulunmasina katkıda bulunan Prof. Dr. Neslihan BAŞÇIL TÜTÜNCÜ'ye sonsuz teşekkürlerimizi sunarız.

\section{KAYNAKLAR}

1. Pekcan G. Beslenme Durumunun Saptanması, Hacettepe Üniversitesi Sağllk Bilimleri Fakültesi Beslenme ve Diyetetik Bölümü, Sağlık Bakanlığı yayım no:726, Klasmat matbaacıllk, Ankara, 2008.

2. DeLegge MH, Guenter P. Energy. In: Gottschlich MM, editor. The A.S.P.E.N Nutrition Support Core Curriculum: A Case-Based Approach: The Adult Patient. USA, Silver Spring: 2007. p.19-29. 
3. Psota T, Chen KY. Measuring energy expenditure in clinical populations: rewards and challenges. Eur J Clin Nutr. 2013;67:436-42.

4. Breen H, Ireton-Jones C. Predicting energy needs in obese patients. Nutr Clin Pract. 2004;19:284-9.

5. Harris JA, Benedict FG. A biometric study of basal metabolism in man. Proc Natl Acad Sci USA. 1918;4(12): 370-3.

6. Da Rocha EE, Alves VG, Silva MH, Chiesa CA, da Fonseca RB. Can measured resting energy expenditure be estimated by formula in daily clinical nutrition practice? Curr Opin Clin Nutr Metab Care. 2005;8:319-28.

7. Wang Z, Heshka S, Zhang K, Boozer CN, Heymsfield SB. Resting energy expenditure: systematic organization and critique of prediction methods. Obes Reas. 2001;9:331-6.

8. Anbar R, Beloosesky Y, Cohen J, Madar Z, Weiss A, Theilla M, et al. Tight calorie control in geriatric patients following hip fracture decreases complications: A randomized, controlled study. Clin Nutr. 2014;33:23-8.

9. Von Pettenkofer M. Ueber einen neuen Respirations apparat. [On a new device for respiration analyses.] 1st ed. Munich, K Akademie in Commission, 1861.

10. Atawer WO, Rosa EB. Description of neo respiration calorimeter and experiments on the conservation of energy in the human body. Vol:36, Washington, DC, Government Printing Office, 1899.

11. Frankenfield DC. On heat, respiration, and calorimetry. Nutrition. 2010;26:339-50.

12. Schadewaldt P, Nowotny B, Straßburger K, Kotzka $\mathrm{J}$, Roden M. Indirect calorimetry in humans: a postcalorimetric evaluation procedure for correction of metabolic monitor variability. Am J Clin Nutr. 2013;97:763-73.

13. Frankenfield D, Roth-Yousey L, Compher C. Comparison of predictive equations for resting metabolic rate in healthy nonobese and obese adults: a systematic review. J Am Diet Assoc. 2005;105:775-89.

14. Henry CJ. Basal metabolic rate studies in humans: measurement and development of new equations. Public Health Nutr. 2005;8:1133-52.

15. da Rocha EEM, Alves VGF, Fonsenca RBV. Indirect calorimetry: methodology, instruments and clinical application. Curr Opin Clin Nutr Metab Care. 2006;9:24756.

16. Schoeller DA. Making indirect calorimetry a gold standard for predicting energy requirements for institutionalized patients. J Am Diet Assoc. 2007;107:3902.

17. Melanson EL, Ingebrigtsen JP, Bergouignan A, Ohkawara K, Kohrt WM, Lighton JR. A new approach for flow- through respirometry measurements in humans. Am J Physiol Regul Integr Comp Physiol. 2010;298:1571-9.

18. Chung-Kang T, Hua-Shui H, Chih-Te H, Hui-Ying H, ChiuShong L, Cheng-Chieh L, et al. Predictive equation of resting energy expenditure in obese adult Taiwanese. ORCP. 2011;5:313-9.

19. Jesus P, Achamrah N, Grigioni S, Charles J, Rimbert A, Folope V, et al. Validity of predictive equations for resting energy expenditure according to the body mass index in a population of 1726 patients followed in a Nutrition Unit. Clin Nutr. 2015;34:529-35.

20. Alves VGF, da Rocha EEM, Gonzalez MC, da Fonseca RBV, do Nascimento Silva MH, Chiesa CA. Assessment of resting energy expenditure of obese patients: Comparison of indirect calorimetry with formulae. Clin Nutr. 2009;28:299-304.

21. Boullata J, Williams J, Cottrell F, Hudson L, Compher C. Accurate determination of energy needs in hospitalized patients. J Am Diet Assoc. 2007;107:393-401.

22. Frankenfield DC, Ashcraft CM, Galvan DA. Prediction of resting metabolic rate in critically ill patients at the extremes of body mass index. JPEN J Parenter Enteral Nutr. 2013;37:361-7.

23. De Waele, Opsomer T, Honoré PM, Diltoer M, Mattens S, Huyghens L, et al. Measured versus calculated resting energy expenditure in critically ill adult patients. Do mathematics match the gold Standard. Minerva Anestesiol. 2015;81:272-82.

24. Daly JM, Heymsfield SB, Head CA, Harvey LP, Nixon DW, Katzeff $\mathrm{H}$, et al. Human energy requirements: Overestimation by widely used prediction equations. Am J Clin Nutr. 1985;42:1170-4.

25. Compher C, Frankenfield D, Keim N, Roth-Yousey L. Evidence Analysis Working Group. Best practice methods to apply to measurement of resting metabolic rate in adults: A systematic review. J Am Diet Assoc. 2006;106:881-903.

26. Alberda C, Snowden L, McCargar L, Gramlich L. Energy requirements in critically ill patients: how close are our estimates? Nutr Clin Pract. 2002;17:38-42.

27. Malone AM. Methods of assessing energy expenditure in the intensive care unit. Nutr Clin Pract. 2002;17:21-8.

28. Wouters-Adriaens MP, Westerterp KR. Low resting energy expenditure in Asians can be attributed to body composition. Obesity (Silver Spring). 2008;16:2212-6.

29. Rodrigues JCD, Lamarca F, de Oliveira CL, Cuppari L, Lourenço LA, Avesania CM. Agreement between prediction equations and indirect calorimetry to estimate resting energy expenditure in elderly patients on hemodialysis. eSPEN Journal. 2014; 9(2):91-6.

30. Nagano A, Yamada Y, Miyake H, Domen K, Koyama T. 
Comparisons of predictive equations for resting energy expenditure in patients with cerebral infarct during acute care. J Stroke Cerebrovasc Dis. 2015;24:1879-85.

31. Ireton-Jones C, Jones JD. Improved equations for predicting energy expenditure in patients: The IretonJones equations. Nutr Clin Pract. 2002;17(1):29-31.

32. Nelson KM, Weinsier RL, Long CL, Schutz Y. Prediction of resting energy expenditure from fat-free mass and fat mass. Am J Clin Nutr. 1992;56(5):848-56.

33. Schofield WN. Predicting basal metabolic rate, new standards and review of previous work. Hum Nutr Clin Nutr. 1985;39:5-41.

34. Harris JA, Benedict FG. A biometric study of human basal metabolism. Proc Natl Acad Sci USA. 1918;4:370-3.

35. Cunningham JJ. Body composition as a determinant of energy expenditure: a synthetic review and a proposed general prediction equation. Am J Clin Nutr. 1991;54:963-9.

36. Siervo M, Bertoli S, Battezzati A, Wells JC, Lara J, Ferraris C, et al. Accuracy of predictive equations for the measurement of resting energy expenditure in older subjects. Clin Nutr. 2014;33(4):613-9.

37. Anderegg BA, Worrall C, Barbour E, Simpson KN, DeLegge M. Comparison of resting energy expenditure prediction methods with measured resting energy expenditure in obese, hospitalized adults. JPEN, J Parenter Enteral Nutr. 2009;33(2):168-75. 